\title{
Cognitive Virtual-Reality Based Stroke Rehabilitation
}

\author{
K. Eng ${ }^{1}$, E. Siekierka ${ }^{2}$, M. Cameirao ${ }^{1}$, L. Zimmerli ${ }^{1}$, P. Pyk ${ }^{1}$, \\ A. Duff ${ }^{1}$, F. Erol ${ }^{1}$, C. Schuster ${ }^{3}$, C. Bassetti ${ }^{2}$, D. Kiper ${ }^{1}$ and P. Verschure ${ }^{1,4}$ \\ ${ }^{1}$ Institute of Neuroinformatics, University/ETH Zurich, Switzerland \\ ${ }^{2}$ Department of Neurology, University Hospital Zurich, Switzerland \\ ${ }^{3}$ Rehaklinik Rheinfelden, Rheinfelden, Switzerland \\ ${ }^{4}$ ICREA and Technology Department, Universitat Pompeu Fabra, Barcelona, Spain
}

\begin{abstract}
Stroke is a debilitating condition with major costs for patients and their care-givers. Here we present a novel virtual reality (VR) based cognitive neurorehabilitation system for improving the rehabilitation of stroke patients with arm and hand paresis. Using a custom, low-cost kinematic tracking system designed for clinical or home use, patients engage in task-oriented interactions with objects in a virtual environment. Our paradigm is based on the hypothesis that observed actions correlated with self-generated or intended actions activate the motor pathways by means of the so-called "mirror-system". This cognitive approach engages cortical motor observation, planning and execution areas. Combined with intensive, task-oriented acute-phase training (1-6 weeks after stroke) when the factors for facilitating recovery are at their highest levels, we postulate that our approach will facilitate cortical plasticity and improve recovery of upper limb function. The tasks range from simple (hitting moving virtual objects) to complex (grasping and moving virtual objects). In the first-person VR environment the patient controls a displayed pair of upper limbs that follow the position and movements of his/her own arms and hands while performing the tasks. The therapist can adjust the contribution of the nonparetic hand in "assisting" the representation of the paretic hand to varying degrees in performing the tasks, simultaneously stimulating the action recognition systems and increasing patient motivation through graded task success. In this paper we present the neuroscientific background of the system, a technical description of its components, and results from testing on healthy subjects and stroke patients.
\end{abstract}

Keywords-Stroke, virtual reality, cognitive therapy

\section{INTRODUCTION}

\section{A. Background}

Stroke can cause a wide range of neurological impairments which severely reduce patient ability to perform activities of daily life (ADL). Severe upper limb paresis has a particularly severe impact on ADL performance and thus on independent living. The prognosis for severe upper limb paresis is poor, with about $30 \%$ not regaining significant dexterity after 6 months $[1,2]$. Current therapy techniques are dominated by occupational and physical therapy, which focus on guided limb manipulation and task-oriented exercises. Newer systems employing virtual reality (VR) technology build on this methodology by increasing the range of possible tasks using novel input/output devices, partly automating therapy procedures, and improving patient motivation using quantified, real-time, task-oriented evaluation and reward [3, 4]. Some systems also provide physical assistance with movement and/or simulate haptic feedback [5-12]. However, the literature is equivocal on the value of physical and occupational therapy for sub-acute stroke patients using conventional or VR-based methods.

Besides its potential to trigger external stimulation, we hypothesize that VR additionally induces use-dependent plastic changes in response to internal stimulation of highermotor cortical areas recruiting the motor "memory" system consisting of stored motor programs. This so-called VRbased interactive cognitive intervention is based on the idea that stimulation of the action processing system in turn activates downstream cortical areas involved in movement execution. Here, a population of specialised neurons, "mirror neurons", plays a key role since these neurons discharge during both action execution and action observation or imagery [13]. With the recruitment of a widespread movement network normally being involved in movement execution, VR-based cognitive therapy offers a potential of specifically promoting/enhancing functional movement recovery.

\section{B. Mirror Neurons}

Mirror neurons, first described in monkey ventral premotor cortex (area 5), discharge when an animal performs a goal-directed hand action and also when it observes another individual performing a similar action. It has been proposed that mirror neurons constitute a vocabulary of hand actions [14]. Their activation leads to recruitment of functionally interconnected cortical structures coupling action execution and observation. More recently, the execution-observation system has also been found in humans [15-17], and there is even evidence that action observation may also induce cortical plasticity [18]. Using Ramachandran's work on pa- 
tients with phantom limbs [19], Altschuler performed a study in chronic stroke patients with a mirror in which they observed their non-paretic (ipsilesional) arm moving, giving them the impression of moving their paretic (contralesional) arm [20]. In a rehabilitation setting, it may thus be possible for a system capable of appropriately stimulating the action observation system to encourage plasticity and repair. Further work showed mirror-like visuomotor activity in humans during object-related hand actions in the posterior parietal lobe [15]. Since task-oriented rehabilitation is known to be beneficial [21], it thus suggests that VR-based cognitive therapy may induce cortical plasticity and promote recovery using goal-directed arm/hand movements.

In addition to action execution and observation, mirror neurons and motor planning areas are known to be activated during voluntary mental motor imagery. Motor imagery selectively modulates muscle excitability [22] - a result used in the training of athletes. Recent studies have also applied motor imagery in stroke rehabilitation, showing potential for cortical reorganization in injured sensorimotor areas [23] and behavioral performance improvements [24].

Overall, these data support the notion that VR-based interactive cognitive therapy based on motor imagery and movement observation with intent to move may induce cortical plasticity and functional recovery in acute stroke patients. In the following sections we present our therapy system for stimulating the action recognition system.

\section{Cognitive VIRTUAL REALITY THERAPY SYSTEM}

The core of the therapy system (Fig. 2) is the Torque multi-user 3D virtual reality gaming environment (GarageGames, Oregon, USA). Our interactive multimedia system uses low-cost input and output devices to facilitate widespread deployment and home use. In the current prototype we are using modified consumer-grade data gloves (P5 data glove/Essential Reality, New York, USA), digital compasses (Honeywell) for hand input, standard PC speakers for audio output and an LCD monitor for visual output.

The patient is seated at a table facing a screen, with their arms on the table in front of them (Fig. 2). The image on the screen shows two arms in the same orientation and relative position, resting on a flat surface representing the table. The movements of the patient's real arms are transferred to the virtual arms in real time. This close correspondence between the real and virtual arms in terms of position, relative orientation and movement is designed to optimally stimulate the patient to treat the virtual arms as their own during the therapy session. To further increase the illusion of ownership, the colour of the sleeves on the virtual arms can be set to match the clothes that the patient is wearing.

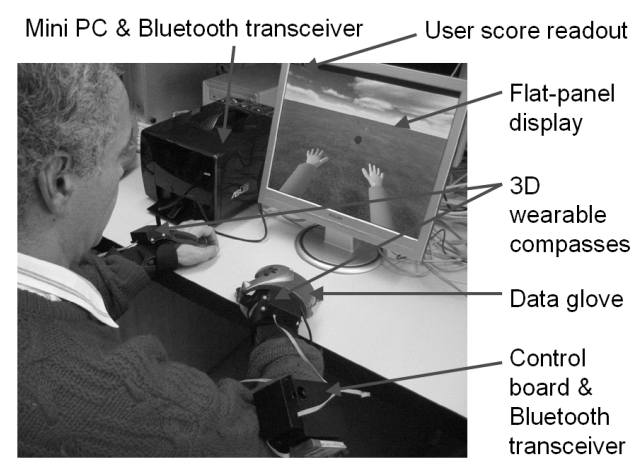

Fig. 1 Overview of cognitive virtual reality therapy system.

The mapping of the real left/right limb pose $\left(P_{L}, P_{R}\right)$ on to the virtual limb pose $\left(P_{V L}, P_{V R}\right)$ is set via four parameters $\alpha_{L L}, \alpha_{R L}, \alpha_{L R}, \alpha_{R R}$, using a mapping function $f$ (Fig. 1):

$$
\left\{\begin{array}{c}
P_{V L} \\
P_{V R}
\end{array}\right\}=\left\{\begin{array}{l}
f\left(P_{L}, \alpha_{L L}, \alpha_{R L}\right) \\
f\left(P_{L}, \alpha_{L R}, \alpha_{R R}\right.
\end{array}\right\}
$$

This mapping enables a variety of control scenarios to be supported, e.g. a patient with a paretic right limb may benefit if the real left limb assists with moving the virtual right limb - a generalization of mirror therapy [20] - to enable easier task success and thus more positive reinforcement.

Table 1 lists some commonly used parameter settings for a linear mapping function $f$. Non-linear mapping functions may be used to deal with boundary conditions related to movement range, task-oriented "assistance" of movements (e.g. snapping towards nearby target objects) and smoothing of jerky movements caused by tremors. Using the left/right mappings, the control of the movements of the "mirrored" arm can be gradually shifted from the intact arm to the paretic arm as the patient recovers, possibly accelerating further the speed of recovery.

It is important that patients try to imitate the movements they see on the screen, even if they cannot move or if the current control mapping does not require it. It is this observation with intent to imitate that we hypothesize to be the optimal way of stimulating the action recognition system.

Table 1 Sample parameter settings for linear mapping function

\begin{tabular}{|c|c|c|}
\hline $\begin{array}{c}\text { Parameter values } \\
\left(\boldsymbol{\alpha}_{\mathrm{LL}}, \boldsymbol{\alpha}_{\mathrm{RL}}, \boldsymbol{\alpha}_{\mathrm{LR}}, \boldsymbol{\alpha}_{\mathrm{RR}}\right)\end{array}$ & $\begin{array}{l}\text { Short } \\
\text { name }\end{array}$ & Description \\
\hline$(1,0,0,1)$ & Normal & Normal 1:1 limb mapping \\
\hline$(1,0,1,0)$ & $\begin{array}{l}\text { Follow } \\
\text { left }\end{array}$ & $\begin{array}{l}\text { Left limb controls movements of } \\
\text { virtual right limb }\end{array}$ \\
\hline$(1,0,-1,0)$ & $\begin{array}{l}\text { Mirror } \\
\text { left }\end{array}$ & $\begin{array}{l}\text { Left limb controls virtual right limb, } \\
\text { movements mirrored about center line }\end{array}$ \\
\hline$(2,0,0,2)$ & Boost & $\begin{array}{l}\text { Virtual left/right limbs move twice as } \\
\text { far as real limbs }\end{array}$ \\
\hline
\end{tabular}


The rehabilitation scenarios provide a graded, personalised training programme of reaching and grasping exercises. The initial scenarios we are testing are:

1. Hitting: the patient must intercept virtual balls moving along the table towards them. Adjustable parameters are: ball speed, interval between successive balls, lateral left/right dispersion of ball start positions and the probability distribution of the ball start positions.

2. Catching: as for Hitting, with the additional constraint that the patient must "catch" the balls by holding the relevant hand open for the ball intercept and closing it within a certain interval after the ball has been intercepted; otherwise it is registered as a "drop". Additional parameters specify the time within which patients must close their hands to register a "catch" event.

3. Grasping: as for Catching, but after catching the ball the patient must move the ball to a movable target location and then release it. Partial success in each phase (intercept, catch, release) scores partial points.

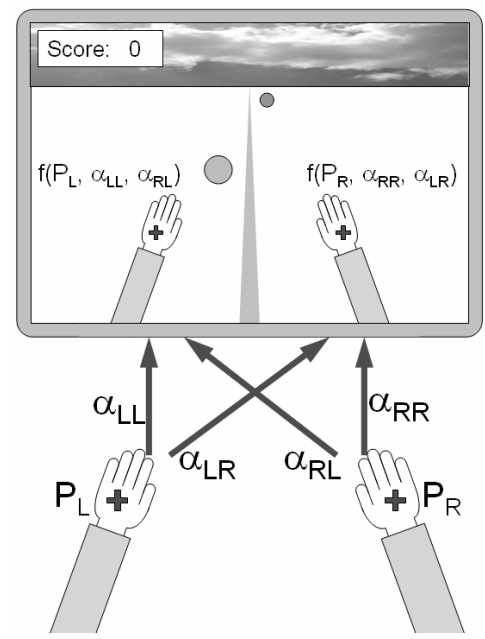

Fig. 2 Schematic of application of weighting factors from real to virtual space. $\mathrm{P}=$ hand pose; $\alpha=$ weighting factors, $\mathrm{f}=$ mapping function. Crosses indicate hand anchor points to which the mapping function is applied.

\section{Pilot Study}

A pilot study is in progress at the Neurology Department of the University Hospital Zurich to test the efficacy of the therapy system on acute stroke patients. The overall goal of the initial study is to assess whether the system can provide additional patient benefits, rather than to replace existing therapies. All procedures have been approved by the responsible institutional ethics committees.

Volunteer patients with moderate to severe hand paresis meeting the entrance criteria (first ever stroke, cortical stroke only, etc.) are admitted into the trial during the first week after stroke onset. After an initial clinical and functional assessment, patients are randomly allocated to either the experimental group or the control group. Both the experimental and control patients receive medical treatment and standard physiotherapy consisting of daily basal taskand ADL-oriented physical therapy in several modules. The experimental group receives, in addition, cognitive VR therapy carried out during one 45-minute session per day on 5 days per week over 5 weeks. The therapy parameters are set by the therapist for each patient individually to maximise motivation by maximising difficulty while keeping trial scores relatively high. All patients are assessed weekly during the treatment phase with an ADL-oriented score (Chedoke Arm \& Hand Activity Inventory [25]) and after 3 months post-stroke to gauge the short-term and mediumterm effectiveness of the system.

\section{Results}

To date, the VR cognitive therapy system has been tested with the hitting scenario on the following subject groups:

- Healthy subjects, age 25-36 years $(\mathrm{N}=19)$, usability

- Acute stroke patients, age 56-63 years $(\mathrm{N}=3)$, usability \& assessment pilot

- Children's hospital patients with various conditions, age 7-14 years $(\mathrm{N}=5)$, usability

All subjects quickly learned to use the system to perform the task during the first session. For the initial settings (1 ball every $2.5 \mathrm{~s}$, slow speed), most subjects were able to intercept $70-100 \%$ of the balls. They had more difficulty with different mapping parameter values (following, mirroring). User acceptance of the system was high (anecdotal and questionnaire responses); in particular the patients expressed a desire to use the system on an ongoing basis. Children were more demanding than adults, requesting various improvements (background music, better graphics).

The upper graph in Fig. 3 shows data from one trial with a right-handed patient with left hemiparesis, with the fates of each of the balls (caught with left hand, caught with right hand, or missed). Most of the "miss" events occurred at the extremities of movement, and a greater proportion of the balls were missed on the left side than the right side. The relative contribution of paresis and handedness to the left/right performance imbalance can only be judged after longitudinal intra-individual testing during the course of recovery as well as from inter-individual comparisons. In the lower graphs the improvement in performance of the patient over multiple sessions can be seen, with the difficulty (speed, rate of ball appearance) increasing steadily while the overall score remains constant. 

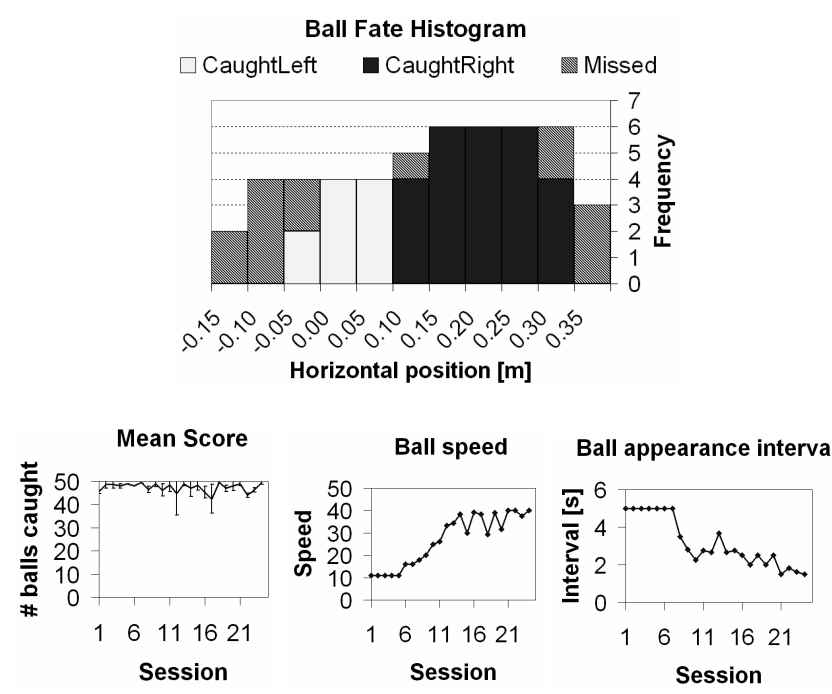

Fig. 3: (Top) Sample histogram of patient performance (hit/missed balls) during a single trial of the Hitting therapy scenario. Each trial consisted of 50 balls. (Bottom) Improvement of an acute stroke patient over time as measured by increase in scenario difficulty while maintaining constant performance. Each session consisted of 4-6 trials.

\section{Conclusions \& Outlook}

We believe that our cognitive stroke rehabilitation method combining action observation with goal-directed movement imagery has significant promise for providing effective rehabilitation based on well-founded neuroscientific hypotheses. In future work we will collect further clinical and basic science data to investigate our claims.

\section{ACKNOWLEDGMENT}

This project is supported by the Swiss National Centre of Competence in Research in Neural Plasticity and Repair, the Gebert Rüf Foundation and Rehatec/DeRoyal.

\section{REFERENCES}

[1] H. Nakayama, H. S. Jorgensen, H. O. Raaschou, and T. S. Olsen, Recovery of upper extremity function in stroke patients: the Copenhagen Stroke Study, Arch. Phys. Med. Rehabil., vol. 75, pp. 394-398, 1994.

[2] G. Kwakkel, B. Kollen, and E. Lindeman, Understanding the pattern of functional recovery after stroke: facts and theories, Restor. Neurol. Neurosci., vol. 22, pp. 281-299, 2004.

[3] M. K. Holden, T. A. Dyer, L. Schwamm, and E. Bizzi, Virtual-EnvironmentBased Telerehabilitation in Patients with Stroke, Presence, vol. 14, pp. 214233, 2005.

[4] M. Holden, Virtual Environments for motor rehabilitation, CyberPsychology \& Behavior, vol. 8, 2005.

[5] S. Jezernik, G. Colombo, T. Keller, H. Frueh, and M. Morari, Robotic Orthosis Lokomat: A Rehabilitation and Research Tool, Neuromodulation, vol. 6, pp. 108-115, 2003.
[6] H. Hummelsheim, M. L. Maier-Loth, and C. Eickhof, The functional value of electrical muscle stimulation for the rehabilitation of the hand in stroke patients, Scand J Rehabil Med, vol. 29, pp. 3-10, 1997.

[7] S. Hesse, H. Schmidt, C. Werner, and A. Bardeleben, Upper and lower extremity robotic devices for rehabilitation and for studying motor control, vol. 16, pp. 705-710, 2003.

[8] B. T. Volpe, M. Ferraro, H. I. Krebs, and N. Hogan, Robotics in the rehabilitation treatment of patients with stroke, vol. 4, pp. 270-276, 2002.

[9] H. I. Krebs, M. Ferraro, S. P. Buerger, M. J. Newbery, A. Makiyama, M. Sandmann, D. Lynch, B. T. Volpe, and N. Hogan, Rehabilitation robotics: pilot trial of a spatial extension for MIT-Manus, vol. 1, pp. 5, 2004.

[10] J. E. Deutsch, J. Latonio, G. C. Burdea, and R. Boian, Post-stroke rehabilitation with the Rutgers Ankle system: a case study, Presence, vol. 10, pp. 416430, 2001.

[11] T. Keller, M. R. Popovic, I. P. Pappas, and P. Y. Muller, Transcutaneous functional electrical stimulator "Compex Motion", vol. 26, pp. 219-223, 2002.

[12] M. R. Popovic and T. Keller, Compex Motion: Neuroprosthesis for grasping applications, in Enabling Technologies: Body Image and Body Function M. MacLachlan and P. Gallagher, Eds.: Elsevier Science Ltd., 2004, pp. 197 -216.

[13] L. Fadiga and L. Craighero, Electrophysiology of action representation, vol. 21, pp. 157-169, 2004

[14] G. Rizzolatti, R. Camarda, L. Fogassi, M. Gentilucci, G. Luppino, and M. Matelli, Functional organization of inferior area 6 in the macaque monkey II. Area F5 and the control of distal movements, Experimental Brain Research, vol. 71, pp. 491-507, 1988

[15] G. Buccino, F. Binkofski, G. R. Fink, L. Fadiga, L. Fogassi, V. Gallese, R. J. Seitz, K. Zilles, G. Rizzolatti, and H.-J. Freund, Action observation activates premotor and parietal areas in a somatotopic manner: an fMRI study, European Journal of Neuroscience, vol. 13, pp. 400-404, 2001.

[16] G. Buccino, F. Binkofski, and L. Riggio, The mirror neuron system and action recognition, Brain and Language, vol. 89, pp. 370-376, 2004.

[17] F. Binkofski, K. Amunts, K. M. Stephan, S. Posse, T. Schormann, H.-J. Freund, K. Zilles, and R. J. Seitz, Broca's region subserves imagery of motion: A combined cytoarchitectonic and fMRI study, Human Brain Mapping, vol. 11, pp. 273-285, 2000.

[18] K. Stefan, L. G. Cohen, J. Duque, R. Mazzocchio, P. Celnik, L. Sawaki, L. Ungerleider, and J. Classen, Formation of a Motor Memory by Action Observation, The Journal of Neuroscience, vol. 25, pp. 9339-9346, 2005.

[19] V. S. Ramachandran and W. Hirstein, The perception of phantom limbs, Brain, vol. 121, pp. 1603-1630, 1998.

[20] E. L. Altschuler, S. B. Wisdom, L. Stone, C. Foster, D. Galasko, D. M. E. Llewellyn, and V. S. Ramachandran, Rehabilitation of hemiparesis after stroke with a mirror, The Lancet, vol. 353, pp. 2035-2036, 1999.

[21] G. Nelles, W. Jentzen, M. Jueptner, S. Muller, and H. C. Diener, Arm training induced brain plasticity in stroke studied with serial positron emission tomography., Neuroimage, vol. 13, pp. 1146-1154, 2001.

[22] L. Fadiga, G. Buccino, L. Craighero, L. Fogassi, V. Gallese, and G. Pavesi, Corticospinal excitability is specifically modulated by motor imagery: a magnetic stimulation study, Neuropsychologia, vol. 37, pp. 147-158, 1999.

[23] S. H. Johnson-Frey, F. R. Maloof, R. Newman-Norhund, C. Farrer, S. Inati, and S. T. Grafton, Actions or Hand-Object Interactions? Human Inferior Frontal Cortex and Action Observation, Neuron, vol. 39, pp. 1053-1058, 2003.

[24] J. A. Stevens and M. E. Stoykov, Using motor imagery in the rehabilitation of hemiparesis, Arch. Phys. Med. Rehabil., vol. 84, pp. 1090-1092, 2003.

[25] S. R. Barreca, P. W. Stratford, C. L. Lambert, L. M. Masters, and D. L. Streiner, Test-Retest Reliability, Validity, and Sensitivity of the Chedoke Arm and Hand Activity Inventory: A New Measure of Upper-Limb Function for Survivors of Stroke, Arch Phys Med Rehabil, vol. 86, pp. 1616-1622, 2005.

Address of the corresponding author:

Author: Kynan Eng

Institute: Institute of Neuroinformatics, Uni/ETH Zurich

Street: Winterthurerstrasse 190

City: $\quad \mathrm{CH}-8057$ Zurich

Country: Switzerland

Email: kynan@ini.phys.ethz.ch 